\title{
Identification of prognosis-related genes in the cervical cancer immune microenvironment
}

\section{Lirong Yang}

Central Laboratory of Yan'an Hospital Affiliated to Kunming Medical University,Key Laboratory of Tumor Immunological Prevention and Treatment of Yunnan Province,Kunming Medical University

\section{Yang Yang}

Central Laboratory of Yan'an Hospital Affiliated to Kunming Medical University,Key Laboratory of Tumor Immunological Prevention and Treatment of Yunnan Province,Kunming Medical University

\section{Mingyao Meng}

Central Laboratory of Yan'an Hospital Affiliated to Kunming Medical University,Key Laboratory of tumor Immunological Prevention and Treatment of Yunnan Province,Yunnan Cell Biology and Clinical Translation Research Center

\section{Wenju Wang}

Central Laboratory of Yan'an Hospital Affiliated to Kunming Medical University,Key Laboratory of Tumor Immunological Prevention and Treatment of Yunnan Province,Yunnan Cell Biology and Clinical

Translation Research Center

\section{Shan $\mathrm{He}$}

Central Laboratory of Yan'an Hospital Affiliated to Kunming Medical University,Key Laboratory of Tumor Immunological Prevention and Treatment of Yunnan Province,Yunnan Cell Biology and Clinical

Translation Research Center

\section{Yiyi Zhao}

Central Laboratory of Yan'an Hospital Affiliated to Kunming Medical University,Key Laboratory of Tumor Immunological Prevention and Treatment of Yunnan Province

\section{Hui Gao}

Central Laboratory of Yan'an Hospital Affiliated to Kunming Medical University,Key Laboratory of Tumor Immunological Prevention and Treatment of Yunnan Province

\section{Weiwei Tang}

Central Laboratory of Yan'an Hospital Affiliated to Kunming Medical University,Key Laboratory of Tumor Immunological Prevention and Treatment of Yunnan Province

\section{Lin Li ( $\nabla$ aileenali@163.com )}

Central Laboratory of Yan'an Hospital Affiliated to Kunming Medical University,Key Laboratory of Tumor Immunological Prevention and Treatment of Yunnan Province,Yunnan Cell Biology and Clinical

Translation Research Center

\section{Zongliu Hou ( hzl579@163.com )}

Yan'an Hospital Affiliated to Kunming Medical University https://orcid.org/0000-0003-3536-7550 


\section{Research article}

Keywords: Cervical cancer, Immune microenvironment, Bioinformatics analysis, Overall survival

Posted Date: March 5th, 2020

DOI: https://doi.org/10.21203/rs.3.rs-16157/v1

License: (1) This work is licensed under a Creative Commons Attribution 4.0 International License. Read Full License

Version of Record: A version of this preprint was published at Gene on January 1st, 2021. See the published version at https://doi.org/10.1016/j.gene.2020.145119. 


\section{Abstract}

Background Cervical cancer is the fourth most common cancer in women worldwide. The metastasis and invasion of this type of cancer are closely related to the tumor microenvironment. Immune cells and stromal cells dominate the tumor microenvironment in cervical cancer. Therefore, we should further understand the association between tumor progress and immune cells or stromal cells.

Methods we downloaded the gene expression profiles and clinical data of 307 patients with cervical cancers based on the TCGA database. Subsequently the Estimation of Stromal and Immune cells in Malignant Tumours using Expression data (ESTIMATE) algorithm was used to calculate the scores of stromal cells and immune cells to find differential genes, and analyzed the correlation between their scores and patient survival. Moreover, we also used R language packs and network tools to analyze GO term, gene enrichment pathway, and protein-protein relationship to find genes related to inflammation and immune regulation.

Results The gene expression profiles and corresponding clinical data of 307 patients were obtained from TCGA datasets. The results showed that there was a statistically significant difference between the high immunescore group and the low immunescore group. And the low immunescore group had shorter lifetimes than the high scores group $(P=0.035)$. Moreover, PPI network analysis CCR5 and CXCL9, $-10,-11$ / CXCR3 axis might be new target for cervical cancer treatment. Finally, Kaplan-Meier survival curves found out nine representative genes significantly related to survival including BTNL8 , CCR7 , CD1E , CD6 , CD27 , CD79A , GRAP2 , SP1B , LY9 .

Conclusions These genes can be used as markers for the prognosis and diagnosis of cervical cancer and also might be used as treatment targets.

\section{Background}

The incidence and mortality of cervical cancer were ranked fourth in the list of new cases and deaths of common cancers in 2018,with $6.6 \%$ and $7.5 \%$ respectively (1). Especially in Eastern Africa, cervical cancer was the most frequently diagnosed cancer in women. There were many risk factors that influence cause of cervical cancer, such as high-risk human papillomavirus(hrHPV) infection, smoking, health conditions, economic status and sexual partners $(2,3)$. Compared with 2012 statistics, the incidence of cervical cancer in 2015 had decreased by $1.3 \%$ (4), possibly dued to production of HPV vaccine, improvement of living conditions and early screening (5). However, it was still a high-mortality cancer in Asia, accounting for $53.8 \%$ of the world. Therefore, essential measures were still needed to reduce the incidence of cervical cancer.

Immunotherapy for cancer is based on the tumor microenvironment (TME), which improves endogenous immune system to monitor and eliminate tumor cells (6). In addition to traditional surgical treatment, radiotherapy, chemotherapy and corresponding vaccines, cervical cancer immunotherapy has gradually emerged $(7,8)$. TME plays a bad role in immunotherapy to suppress immune cell function and promote 
tumor cell infiltration (9). TME is mainly composed of cellular and molecular parts, including fibroblasts, endothelial cells, tumor cells, extracellular matrix (ECM), immune cells and vasculature (10). ESTIMATE algorithm, which is based on sample Gene Set Enrichment Analysis, create immune and stromal score to predict their respective infiltration level in tumor (11). Currently, several cancer types had been evaluated the infiltration results in TME through using ESTIMATE algorithm(12-15). However, as far as we known, there was no report to systematically assess the infiltration condition in TME of cervical cancer yet.

Therefore, in the present study, we would estimate immune and stromal score in TME based on cervical cancer data obtained from The Cancer Genome Atlas (TCGA) database to further find genes related to the diagnostic and prognosis of cervical cancer.

\section{Methods}

\section{Database}

The gene expression profiles of adult patients with cervical cancer and corresponding patients' clinical profiles dataset including outcome, age, TNM stage and survival time were downloaded from the TCGA database (https://gdc.nci.nih.gov/) (16). Overall stromal content (StromalScore), immune infiltration (ImmuneScore) and the combined (ESTIMATEScore) score of each tumor samples were assessed by the ESTIMATE algorithm of the down-loaded database (17).

\section{Differentially expressed genes (DEG) identification}

All carcinoma of uterine cervix patients were divided into high-score and low-score groups based on median score of the immune and stromal scores. Grouping criteria were based on DEGs with a $p$ value and false discovery rate (FDR) less than 0.05 and fold change greater than 2 times.In this study, package edgeR was used for data analysis and heatmap of the DEGs were drawn using the pheatmap R package.

\section{GO and pathway enrichment analyses}

The R package(18) was applied to analyze DEG functions and KEGG pathway enrichment. GO term analysis consists of biological processes (BP), cellular component (CC), and molecular function (MF). The ClueGo plug-in from Cytoscape software were employed to analyze DEGs enriched in various pathways. Differences of genes between high score group and low score group were considered to be statistically significant at $\mathrm{P}<0.05$ and were reported.

\section{PPI network construction}

The STRING database (http://string-db.org/cgi/input.pl) is an online tool for evaluating protein-protein interaction (PPI) information(19). We used STRING database to assess prepared DEGs-encoded proteins and interactions were selected with an interaction score greater than 0.95. Cytoscape software was applied to establish PPI network, and the Molecular Complex Detection (MCODE) in Cytoscape software 
was subsequently utilized to carry out modular analysis based on the node number and MCODE score. The most significant module was identified according to node number and the MCODE score (20).

\section{Survival analysis}

Kaplan-Meier plots were performed to analyze correlations between the overall survival of patients with carcinoma of uterine cervix and expression of DEGs or immune/stromal scores. The log-rank test was used to calculate statistical significance of the correlation. The survival analyses were performed in TCGA datasets by R"survival" package.

\section{Results}

\section{Immune microenvironment correlates with clinical characteristics of patients with cervical cancer}

The gene expression profiles and corresponding clinical data of 307 patients were obtained from TCGA datasets. The youngest patients were 20 years old, the oldest 88 years old, and the median was 46 years old.

We used the ESTIMATE algorithm to calculate the immunescore ranging from -1209.74 to 3419.323 , stromalscore ranging from -2437.40 to 804.22 and ESTIMATEScore ranging from -3262.05 to 4002 of all patients (13). This data indicated that the higher the ESTIMATEScore, the less the proportion of tumor cells. In order to study the potential relationship between clinical overall survival of patients and their immunescores or stromalscores, we divided the 307 patients into high- and low-score groups according to immunescore or stromalscore, then evaluated their relationship with Kaplan-Meier survival analysis. The results showed that there was a statistically significant difference between the high immunescore group and the low immunescore group. And the low immunescore group had shorter lifetimes than the high scores group $(P=0.035$, Fig. $1 A)$. However, there was no significant difference between the high stromalscore group and low stromalscore group for nearly 15 years. $(P=0.391$, Fig. $1 B)$

Furthermore, according to the clinical TMN stage of the tumor, patients with cervical cancer were classified into two groups: $\mathrm{M} 1$ and $\mathrm{M} 0$ stage. We analyzed the relationship between $\mathrm{M} 1 / \mathrm{M} 0$ stage and their corresponding immune score or stromal score. As shown in Figure 1C,D, the immunescore and stromalscore in $\mathrm{M} 0$ stage group were significantly higher than those in $\mathrm{M} 1$ stage group $(P=0.048, P=0.027)$.

\section{Identification of DEGs based on immunescore and stromalscore}

The expression profile data for all 307 patients with cervical cancer from TCGA was investigated to screen for DEGs between the high score and low score groups. A total of 1067 and 946 DEGs in cervical cancer sample cells based on immunescores and stromalscores, respectively. The heat map for DEGs of immune/stromal score groups was shown that DEGs were classified into 2 clusters. Genes in cluster with 
blue bar were belonged to high score group, while genes in cluster with pink bar were belonged to low score group in cervical cancer samples (Fig. 2A,B). Among them, we identified 408 up-regulated genes and 17 down-regulated genes in Fig. 2C,D from both immunescore groups and stromalscore groups.

\section{GO and KEGG pathway enrichment analyses}

In order to study the enrichment analysis of DEGs, we completed the enrichments of GO, including BP, CC, and MF. KEGG enrichment analysis of DEGs using the R package. DEGs in the BP category were mainly associated with $T$ cell activation, regulation of lymphocyte activation, regulation of $\mathrm{T}$ cell activation and lymphocyte differentiation. For CC, DEGs were frequently enriched in the GO term of external side of plasma membrane, plasma membrane receptor complex, immunological synapse and $\mathrm{T}$ cell receptor complex. With regard to MF, DEGs were primarily enriched in cytokine receptor activity, cytokine receptor binding and immunoglobulin binding (Fig. 3A). Subsequently, the KGEE enrichment analysis of DEGs was completed and found mainly clustered in the cytokine-cytokine receptor interaction, chemokine signaling pathway, cell adhesion molecules and the intestinal immune network for IgA production (Fig.3B,C).

\section{Construction of PPI Network and module analysis}

PPI network of DEGs for cervical cancer was constructed with 150 nodes and 282 edges based on the STRING network tool and Cytoscape software (Fig. 4A). We selected the top twelve DEGs with the number of nodes, including CXCL10(15), CXCL9(14), CCL5 (13), CD4(13), CCL19(12), CCL21(12), CCR5(12), CD3E(12), CXCL11(12), IL10(12), ITGB2(12)and TYROBP(12) (Fig. 4D).

Cytotype MCODE software was used to analyze Clustering analysis of the PPI network based on the above-mentioned PPI network. The two significant modules based on the degree of importance were selected. The module 1 contained 11 nodes and 48 edges, including aforementioned CXCL10, CXCL11, CXCL9, CCL21,CCR5, CCL19, CCL5 (Fig. 4D). Another one, module 2 contained 6 nodes and 15 edges, including CD4, CD3D, CD3E, CD3G, ITK, LCP2(Fig. 4C).

\section{Survival analysis}

We investigated the potential value between DEGs and overall survival in patients with cervical cancer using Kaplan-Meier survival curves. 149 DEGs were significantly correlated with the overall survival of patients among the 425 commonly DEGs according to the log-rank test $(P<0.05)$. As shown in Fig. 5 , several representative genes are represented $(p<0.001)$.

\section{Discussion}

Although the incidence of cervical cancer had generally declined, poor prognosis was most frequently stated problem with advanced and metastatic cervical cancer (21). In recent years, immunotherapy emerged as a novel treatment option, opening the new door to tumor treatment (22). But it still had little effect in solid tumors, including cervical cancer. Mainly because the stromal cells build a complex and hard barrier for the TME, so that immune cells are difficult to infiltrate into the tumor tissue. The 
metastasis and progression of cervical cancer were mainly due to the loss of cancer cell adhesion and the degradation of the extracellular matrix leading to the invasion phenotype of the cells from in situ metastasis. Moreover, tumor evoluted a series of ways to escape immune surveillance even facing continuous immune pressure in the $\operatorname{TME}(23,24)$. Given stromal cells and immune cells played pivotal role in the cervical cancer microenvironment. It was essential to further elucidate the their correlation between the survival of patientsand immune cells or stromal cells in cervical cancer.

In this study, we downloaded the gene expression profile and clinical database of cervical cancer from the TCGA database using a web tool to study the composition of cervical cancer TME including stromal cells and immune cells through ESTIMATE algorithm for the first time. Meanwhile, the scores of stromal cells and immune cells calculated with ESTIMATE algorithm, then their correlation with the survival of cervical cancer patients were also analyzed. Finally, we found DEGs correlation using GO analysis, signaling pathway enrichment, PPI network construction and compared the relationship between DEGs and overall survival by package edgeR.

At present, few studies explored the relationship between the prognosis of cervical cancer and the infiltration condition of immune cells or stromal cells. In this study, the ESTIMATE algorithm was used to calculate the scores of immune cells or stromal cells, which were divided into high score group and low score group. The calculated results demonstrated that these scores were strongly associated with the survival in cervical cancer. Some previous studies had found that in cervical cancer patients with highrisk, immune-related genes might reflect a changing tumor microenvironment (25). Unbalanced activation of Th17 and regulatory T cells might contribute to cancer progression and had a poorer prognosis (26). Th17 cells differentiation signaling pathways in KEGG analysis had also been mentioned in cervical cancer progression. Moreover, intense interaction between the tumor and the surrounding stroma cells could lead to a vicious circle of cancer (27). Based on our experimental data of immune / stromal scores associated with the survival of cervical cancer patients, the results were coincident with others' findings. It was further speculated that the TME or the content of immune cells and stromal cells would have a correlation with the survival of patients with cervical cancer. Various intrinsic factors such as disease stage, lymph node metastasis, and distant metastasis of cervical cancer had been studied to identify factors associated with cancer survival $(28,29)$. Our data also showed that immune/stroma scores were closely related to distant metastasis, which was consistent with their studies.

Subsequently, we found DEGs between high and low score groups, and processed them using bioinformatics methods including GO term analysis, KEGG analysis and PPI network construction. GO term analysis showed that DEGs were mainly enriched in T cell activation, external side of plasma membrane and cytokine receptor activity. KEGG pathway analysis observed DEGs mainly clustered in for the cytokine-cytokine receptor interaction, chemokine signaling pathway, cell adhesion molecules and the intestinal immune network for IgA production. Moreover, according to DEGs of the PPI network, we identified two modules including some chemokines and CD3 molecules which were relevant with the immune and inflammatory response. 
Chemokines are small proteins that binds to the $\mathrm{G}$ protein-coupled receptors. Among them, CXCL9, -10 , -11 are selective ligands for the CXCR3 receptor. CXCL9, CXCL10, CXCL11 / CXCR3 axis played an important role that regulate the migration (espacially chemotactic migration), activation and proliferation of immune cells. On the other hand, it also caused cancer cell metastasis mainly due to tumor-derived CXCR3A ligand activity $(30,31)$. Our present work found that this axis was also expressed in cervical cancer. In addition, through our PPI network analysis chemokine receptor CCR5 was also expressed. The previous study revealed $C D 8^{+} T$ cells expressing CCR5 and CXCR3 chemotactic receptors preferentially proliferated in cervical cancer (32). And anti-CXCR3 treatment could suppress tumor metastasis and tumor-host response. Furthermore, down-regulating the expression of CCR5 inhibited the proliferation and invasion of cervical cancer $(33,34)$. Therefore, CCR5 and CXCL9, -10, -11 / CXCR3 axis might be new target for cervical cancer treatment.

CD3D, CD3G, and CD3E genes are three of the genes encoding CD3 chain in TCR/CD3 complex. Their mutations caused severe immune deficiencies and showed increased susceptibility to infections early(35). It was reported that a high CD3D / CD 4 ratio predicted better survival and also be used as a prognostic marker for bladder cancer(36). Our result also provided the evidence about CD3 / CD4 expression involved in cervical cancer progression

Finally, we verified 9 representative genes including BTNL8, CCR7, SPIB, CD6, LY9, CD1E, CD27, CD79A, GRAP2 as prognostic markers of cervical cancer, which needed further verification in clinical. Among them, CD27 and CCR7 were markers of T cell differentiation. Previous studies showed that advanced

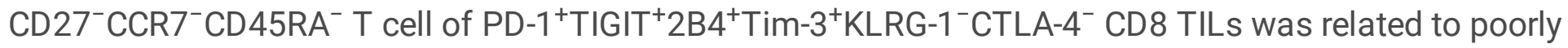
differentiated cervical cancer (37), and CCR7 might be associated with lymph node metastasis. So that they could be used as a prognostic factor for survival (38). As we known, the remaining 7 genes were the first time to be reported to possess relationship with cervical cancer, thus they might also be used as a reference for the prognosis of this type of cancer.

\section{Conclusion}

In conclusion, we used the ESTIMATE algorithm for the first time to evaluate the score of stromal cells and immune cells in cervical cancer based on TCGA database, and analyzed the DEGs between immune cells and stromal cells through systematic bioinformatics analysis. Based on our study, more understanding on complicated relationship between cervical cancer microenvironment and tumors were promoted, as well as some potential biomarkers related to cervical cancer were found. Of course, these molecular markers for the diagnosis and prognosis of cervical cancer still need to be further verified in clinical.

\section{Abbreviations}

ESTIMATE: Estimation of Stromal and Immune cells in Malignant Tumours using Expression data ; hrHPV: high-risk human papillomavirus; TME: Tumor microenvironment; TCGA: The Cancer Genome 
Atlas; DEG: Differentially expressed genes; FDR: false discovery rate; BP: biological processes; CC: cellular component; MF: molecular function; PPI: protein-protein interaction; MCODE: Molecular Complex Detection.

\section{Declarations}

\section{Acknowledgements}

The authors would like to thank Mr. Tao Peng for his technical support.

\section{Authors' contributions}

LRY, ZLH and LL designed the current study. LRY, ZLH and LL performed the analyses and calculations. LRY, LL contributed to the writing of the manuscript, ZLH, LL supervised the study. All authors read and approved the final manuscript.

\section{Funding}

This work was supported by National Natural Science Foundation of China [grant no 81960499 and 81660477] , Science and Technology Department of Yunnan Province [grant no 2017FE468(-007) and 2017NS335], The funding agencies had no bearing

in the design and conduct of the study or the write-up and publication of the study results.

\section{Availability of data and materials}

The datasets generated and/or analyzed during the current study were TCGA

(https://portal.gdc.cancer.gov/). The datasets used and analyzed during the current study are available from the corresponding author on reasonable request.

\section{Ethics approval and consent to participate}

Not applicable.

\section{consent for publication}

Not applicable.

\section{Competing interests}

The authors declare that they have no competing interests.

\section{References}


1. Ferlay J, Colombet M, Soerjomataram I, Mathers C, Parkin DM, Pineros $M$, et al. Estimating the global cancer incidence and mortality in 2018: GLOBOCAN sources and methods. International journal of cancer. 2019;144(8):1941-53.

2. Olusola P, Banerjee HN, Philley JV, Dasgupta S. Human Papilloma Virus-Associated Cervical Cancer and Health Disparities. Cells. 2019;8(6).

3. Gaffney DK, Hashibe M, Kepka D, Maurer KA, Werner TL. Too many women are dying from cervix cancer: Problems and solutions. Gynecologic oncology. 2018;151(3):547-54.

4. Ferlay J, Soerjomataram I, Dikshit R, Eser S, Mathers C, Rebelo M, et al. Cancer incidence and mortality worldwide: sources, methods and major patterns in GLOBOCAN 2012. International journal of cancer. 2015;136(5):E359-86.

5. Vaccarella S, Lortet-Tieulent J, Plummer M, Franceschi S, Bray F. Worldwide trends in cervical cancer incidence: impact of screening against changes in disease risk factors. European journal of cancer. 2013;49(15):3262-73.

6. Mahoney KM, Rennert PD, Freeman GJ. Combination cancer immunotherapy and new immunomodulatory targets. Nature reviews Drug discovery. 2015;14(8):561-84.

7. Wang Q, Steger A, Mahner S, Jeschke U, Heidegger H. The Formation and Therapeutic Update of Tumor-Associated Macrophages in Cervical Cancer. International journal of molecular sciences. 2019;20(13).

8. Ventriglia J, Paciolla I, Pisano C, Cecere SC, Di Napoli M, Tambaro R, et al. Immunotherapy in ovarian, endometrial and cervical cancer: State of the art and future perspectives. Cancer treatment reviews. 2017;59:109-16.

9. Chen YP, Zhang Y, Lv JW, Li YQ, Wang YQ, He QM, et al. Genomic Analysis of Tumor Microenvironment Immune Types across 14 Solid Cancer Types: Immunotherapeutic Implications. Theranostics. 2017;7(14):3585-94.

10. Liu Y, Cao X. Characteristics and Significance of the Pre-metastatic Niche. Cancer cell. 2016;30(5):668-81.

11. Yoshihara K, Shahmoradgoli M, Martinez E, Vegesna R, Kim H, Torres-Garcia W, et al. Inferring tumour purity and stromal and immune cell admixture from expression data. Nature communications. 2013;4:2612.

12. Fang Bai1 YJ, Peng Zhang, Hongliang Chen, Yipeng Fu, Mingdi Zhang, Ziyi Weng,Kejin Wu. Bioinformatic profiling of prognosis-related genes in the breast cancer immune microenvironment. AGING-US. 2019;11.

13. Yan H, Qu J, Cao W, Liu Y, Zheng G, Zhang E, et al. Identification of prognostic genes in the acute myeloid leukemia immune microenvironment based on TCGA data analysis. Cancer immunology, immunotherapy : CII. 2019;68(12):1971-8.

14. Lopez-Doriga A, Valle L, Alonso MH, Ausso S, Closa A, Sanjuan X, et al. Telomere length alterations in microsatellite stable colorectal cancer and association with the immune response. Biochimica et biophysica acta Molecular basis of disease. 2018;1864(9 Pt B):2992-3000. 
15. Lin CY, Kwon H, Rangel Rivera GO, Li X, Chung D, Li Z. Sex Differences in Using Systemic Inflammatory Markers to Prognosticate Patients with Head and Neck Squamous Cell Carcinoma. Cancer epidemiology, biomarkers \& prevention : a publication of the American Association for Cancer Research, cosponsored by the American Society of Preventive Oncology. 2018;27(10):1176-85.

16. Cancer Genome Atlas N. Comprehensive molecular portraits of human breast tumours. Nature. 2012;490(7418):61-70.

17. Yoshihara K VR. Hiding in the dark: uncovering cancer drivers through image-guided genomics. Genome Biol. 2014;15:563.

18. Yu G, Wang LG, Han Y, He QY. clusterProfiler: an R package for comparing biological themes among gene clusters. Omics : a journal of integrative biology. 2012;16(5):284-7.

19. Szklarczyk D, Franceschini A, Wyder S, Forslund K, Heller D, Huerta-Cepas J, et al. STRING v10: protein-protein interaction networks, integrated over the tree of life. Nucleic Acids Research. 2015;43(D1):D447-D52.

20. Gary D Bader CWH. An automated method for finding molecular complexes in large protein interaction networks. BMC Bioinformatics. 2003;4.

21. Islami F, Fedewa SA, Jemal A. Trends in cervical cancer incidence rates by age, race/ethnicity, histological subtype, and stage at diagnosis in the United States. Preventive medicine. 2019;123:31623.

22. Michalis Liontos $\mathbb{A}$ AK, loannis Dimitriadis, Meletios-Athanasios Dimopoulos,Aristotelis Bamias. Systemic therapy in cervical cancer: 30 years in review. CRIT REV ONCOL HEMAT. 2019;137.

23. Yadav SS, Prasad CB, Prasad SB, Pandey LK, Singh S, Pradhan S, et al. Anti-tumor activity of staurosporine in the tumor microenvironment of cervical cancer: An in vitro study. Life sciences. 2015;133:21-8.

24. Piersma SJ. Immunosuppressive tumor microenvironment in cervical cancer patients. Cancer microenvironment : official journal of the International Cancer Microenvironment Society. 2011;4(3):361-75.

25. Yang S, Wu Y, Deng Y, Zhou L, Yang P, Zheng Y, et al. Identification of a prognostic immune signature for cervical cancer to predict survival and response to immune checkpoint inhibitors. Oncoimmunology. 2019;8(12):e1659094.

26. Alves JJP, De Medeiros Fernandes TAA, De Araujo JMG, Cobucci RNO, Lanza DCF, Bezerra FL, et al. Th17 response in patients with cervical cancer. Oncology letters. 2018;16(5):6215-27.

27. De Nola R, Menga A, Castegna A, Loizzi V, Ranieri G, Cicinelli E, et al. The Crowded Crosstalk between Cancer Cells and Stromal Microenvironment in Gynecological Malignancies: Biological Pathways and Therapeutic Implication. International journal of molecular sciences. 2019;20(10).

28. Ittiamornlert P, Ruengkhachorn I. Neutrophil-lymphocyte ratio as a predictor of oncologic outcomes in stage IVB, persistent, or recurrent cervical cancer patients treated by chemotherapy. BMC cancer. 2019;19(1):51. 
29. Heeren AM, Koster BD, Samuels S, Ferns DM, Chondronasiou D, Kenter GG, et al. High and interrelated rates of PD-L1+CD14+ antigen-presenting cells and regulatory $T$ cells mark the microenvironment of metastatic lymph nodes from patients with cervical cancer. Cancer immunology research. 2015;3(1):48-58.

30. Tokunaga R, Zhang W, Naseem M, Puccini A, Berger MD, Soni S, et al. CXCL9, CXCL10, CXCL11/CXCR3 axis for immune activation - A target for novel cancer therapy. Cancer treatment reviews. 2018;63:40-7.

31. Lasagni L, Francalanci M, Annunziato F, Lazzeri E, Giannini S, Cosmi L, et al. An alternatively spliced variant of CXCR3 mediates the inhibition of endothelial cell growth induced by IP-10, Mig, and I-TAC, and acts as functional receptor for platelet factor 4. J Exp Med. 2003;197(11):1537-49.

32. Domingos-Pereira S, Decrausaz L, Derre L, Bobst M, Romero P, Schiller JT, et al. Intravaginal TLR agonists increase local vaccine-specific CD8 T cells and human papillomavirus-associated genitaltumor regression in mice. Mucosal immunology. 2013;6(2):393-404.

33. Che LF, Shao SF, Wang LX. Downregulation of CCR5 inhibits the proliferation and invasion of cervical cancer cells and is regulated by microRNA-107. Experimental and therapeutic medicine. 2016;11(2):503-9.

34. Cambien B, Karimdjee BF, Richard-Fiardo P, Bziouech H, Barthel R, Millet MA, et al. Organ-specific inhibition of metastatic colon carcinoma by CXCR3 antagonism. Br J Cancer. 2009;100(11):1755-64.

35. Alain Fischer GrdSB, Franc, oise Le Deist. CD3 deficiencies. Curr Opin Allergy Clin Immunol. 2005;5.

36. Shi MJ, Meng XY, Wu QJ, Zhou XH. High CD3D/CD4 ratio predicts better survival in muscle-invasive bladder cancer. Cancer management and research. 2019;11:2987-95.

37. Li X, Wang R, Fan P, Yao X, Qin L, Peng Y, et al. A Comprehensive Analysis of Key Immune Checkpoint Receptors on Tumor-Infiltrating T Cells From Multiple Types of Cancer. Frontiers in oncology. 2019;9:1066.

38. Kodama J, Hasengaowa, Kusumoto T, Seki N, Matsuo T, Ojima Y, et al. Association of CXCR4 and CCR7 chemokine receptor expression and lymph node metastasis in human cervical cancer. Annals of oncology : official journal of the European Society for Medical Oncology. 2007;18(1):70-6.

\section{Figures}


A

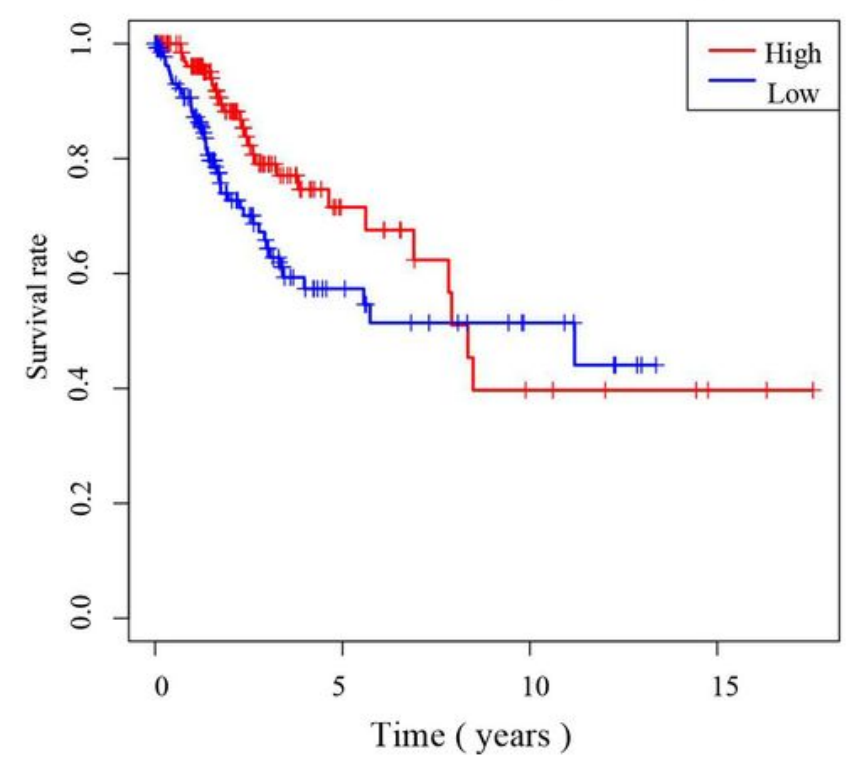

C

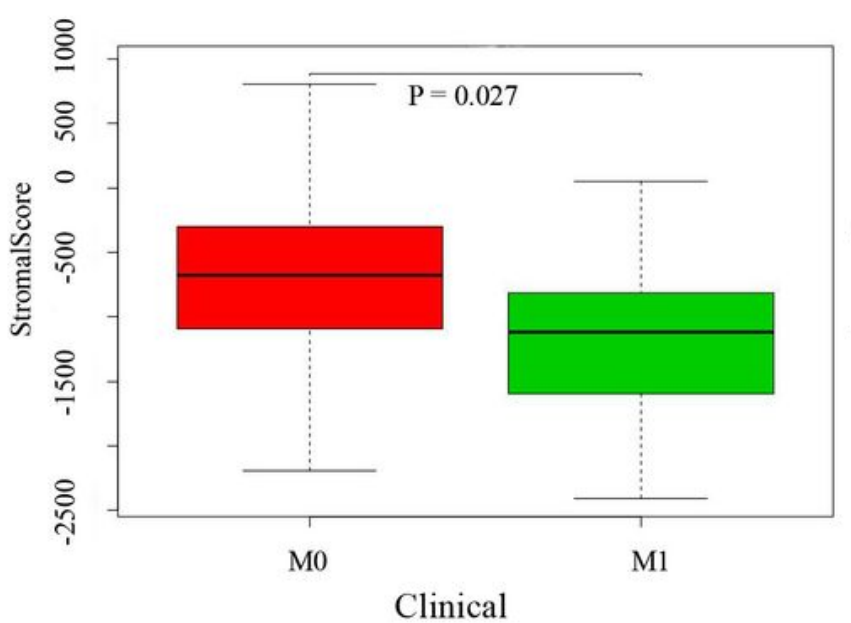

B StromalScore $(\mathrm{P}=0.391)$

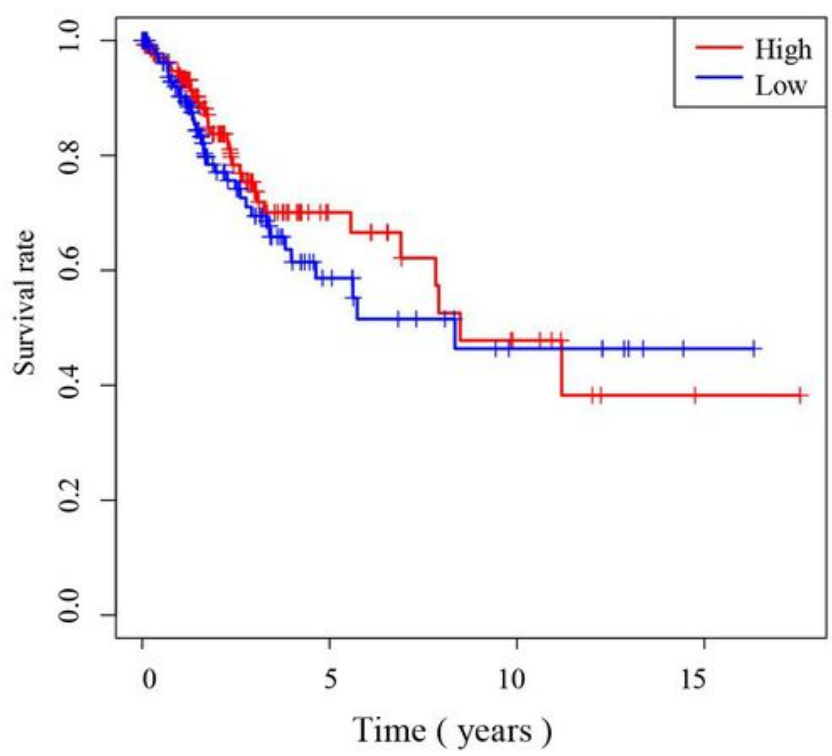

D

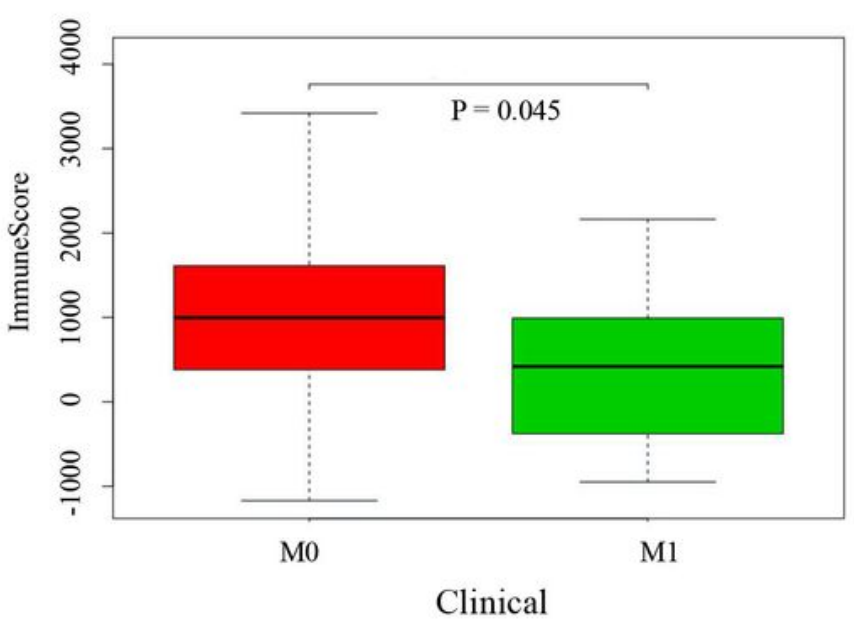

\section{Figure 1}

Immune microenvironment correlates with clinical characteristics of patients. (A) The line chart showed the relationship between immune score and the survival of patients. The high score group had a longer survival time than the low score group. (B) The line chart showed the relationship between stromal score and the survival of patients. (C\&D) Cervical cancer metastasis was significantly related to proportion of immune cells and stromal cells. 


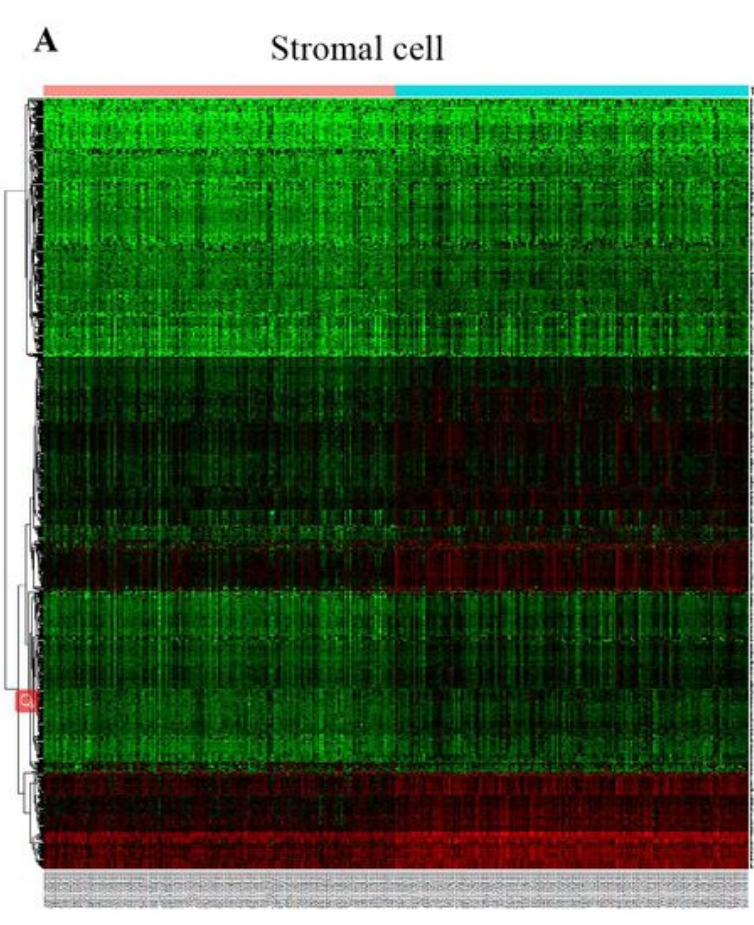

C

Down

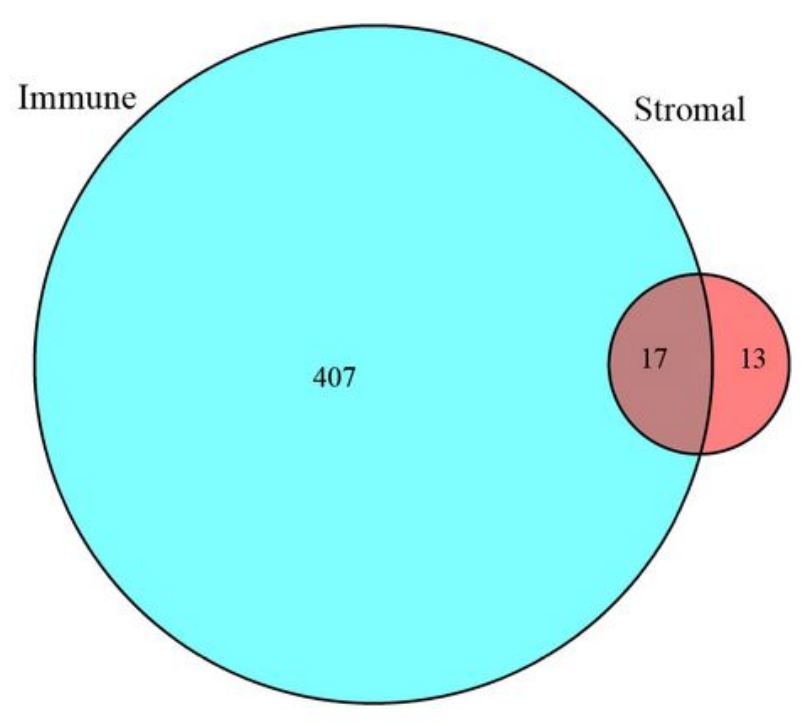

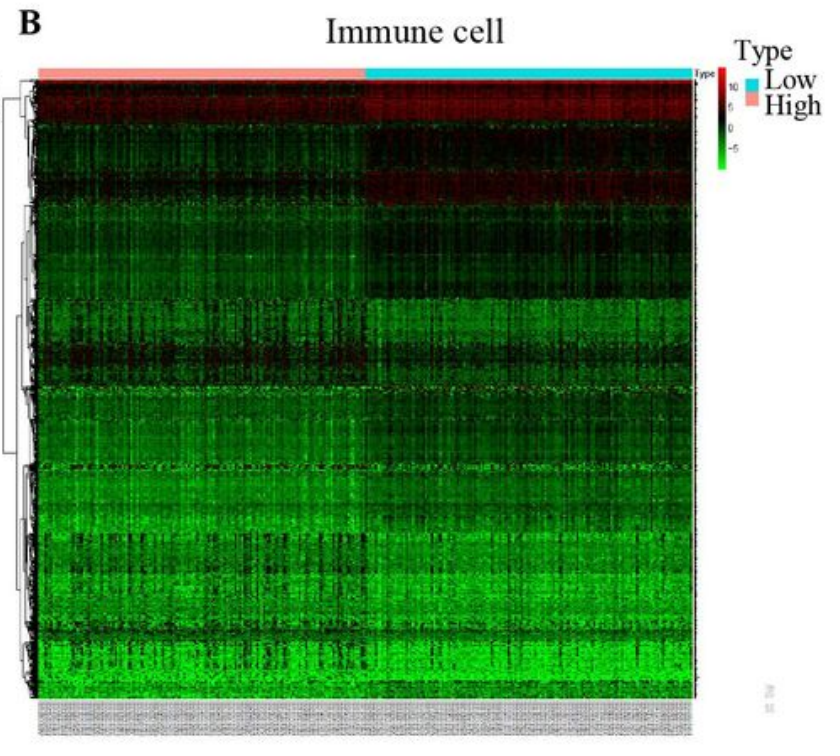

D

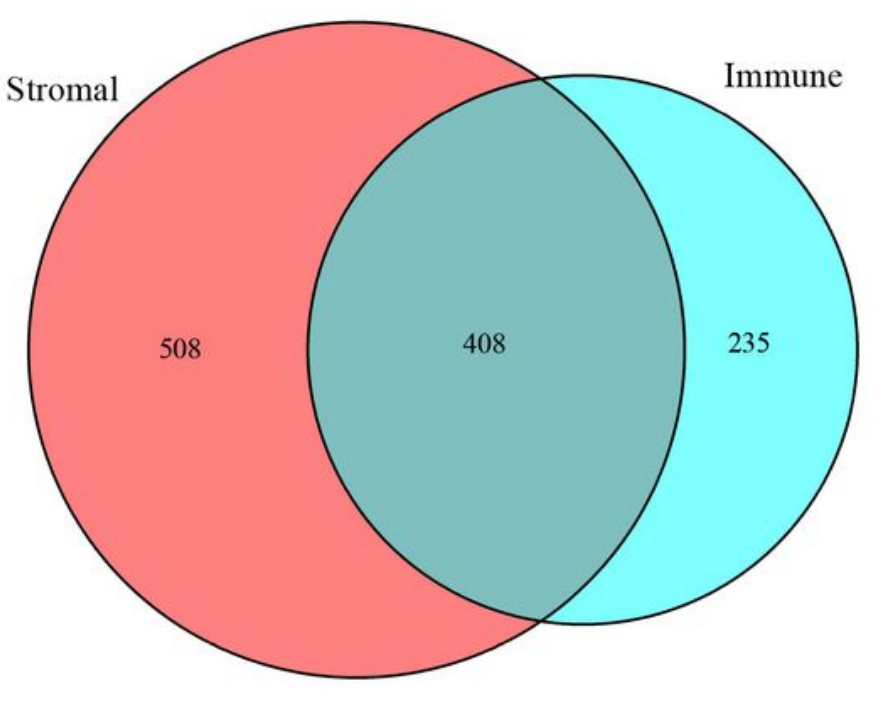

\section{Figure 2}

Identification of DEGs based on immune scores and stromal scores. (A\&B). Heat map of DEGs of immune cells and stromal cells. (C).Common intersection of immune cells and stromal cells downregulating genes. (D). Common intersection of immune cells and stromal cells up-regulating genes. 
A

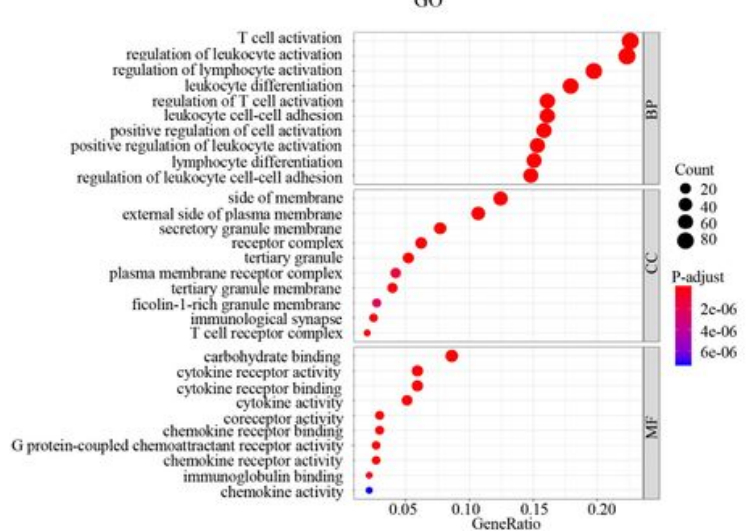

B

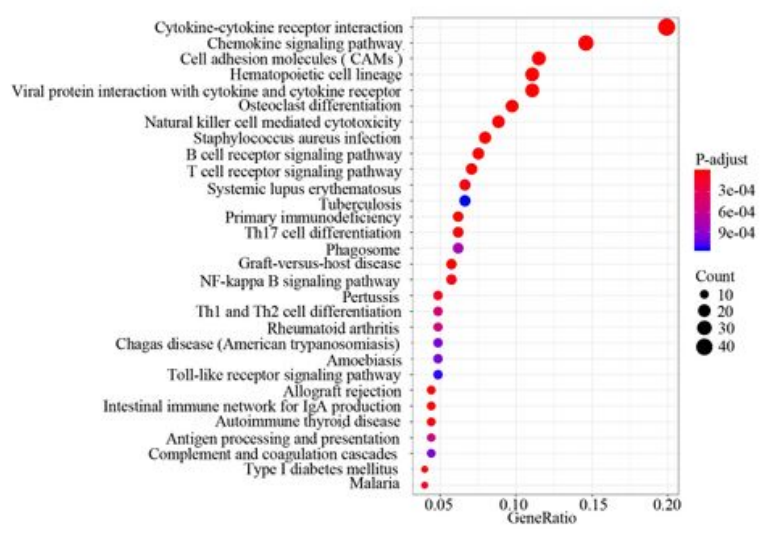

C

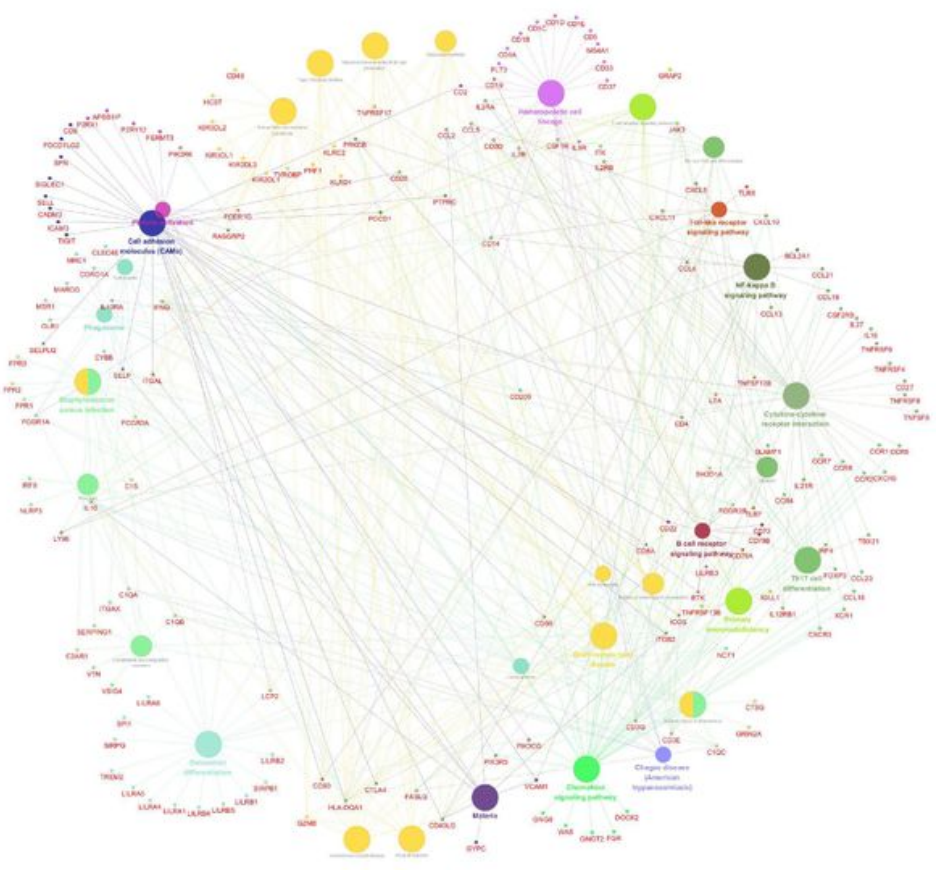

Figure 3

GO and KEGG pathway enrichment analyses. (A). The GO term analysis of 30 enrichments, including biological process, molecular function and cellular component. (B\&C) KEGG pathway analysis confirmed the pathway enrichment of these DEGs and the specific distribution of DEGs in each pathway. 


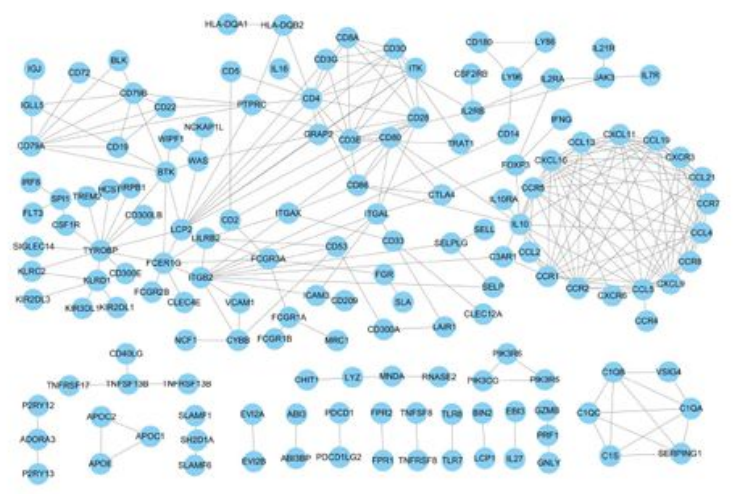

B
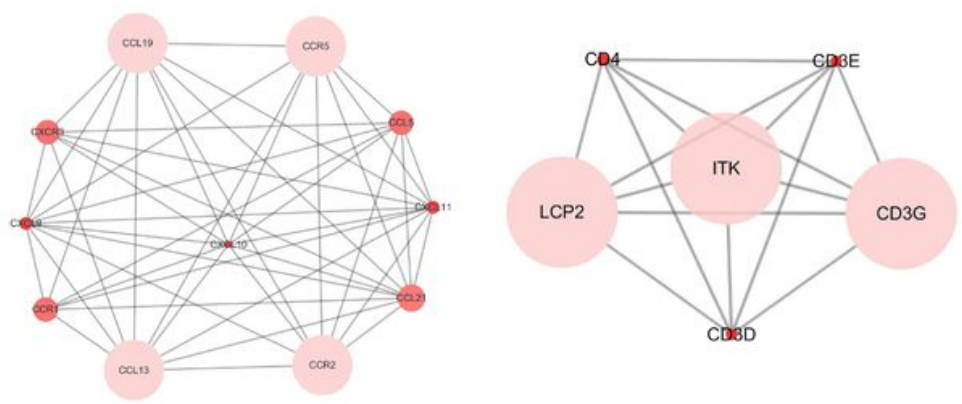

D

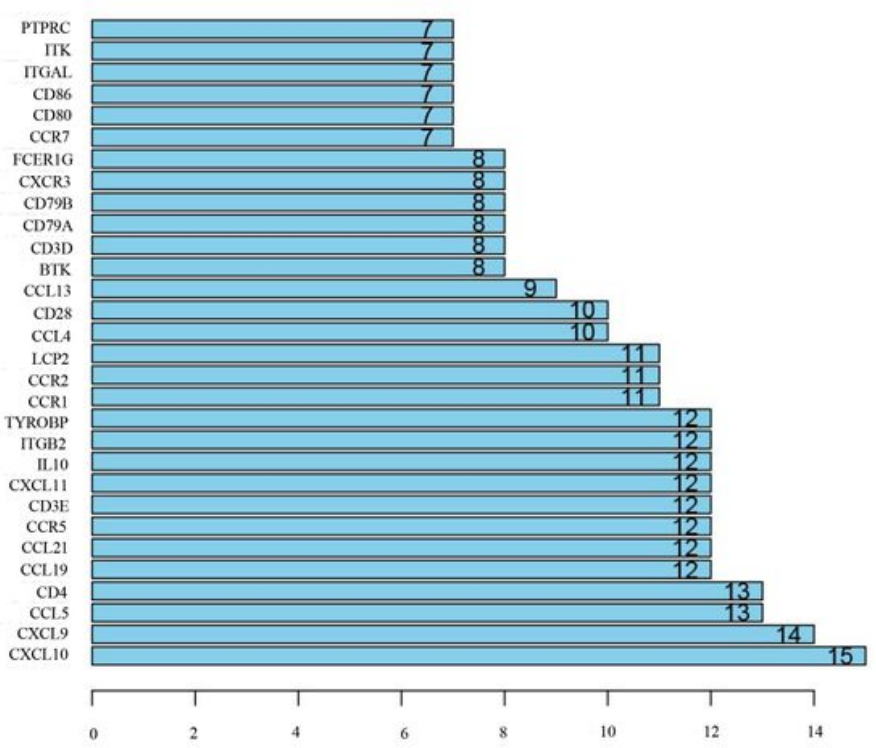

Figure 4

Construction of PPI Network and module analysis. (A). PPI network including 150 nodes and 282 edges was constructed, based on the STRING network tool and Cytoscape software. (B). Two significant modules were selected based on Cytotype MCODE software. The module 1 contained 11 nodes and 48 edges. (C). The module 2 contained 6 nodes and 15 edges. (D). The number of nodes of top twelve DEGs 

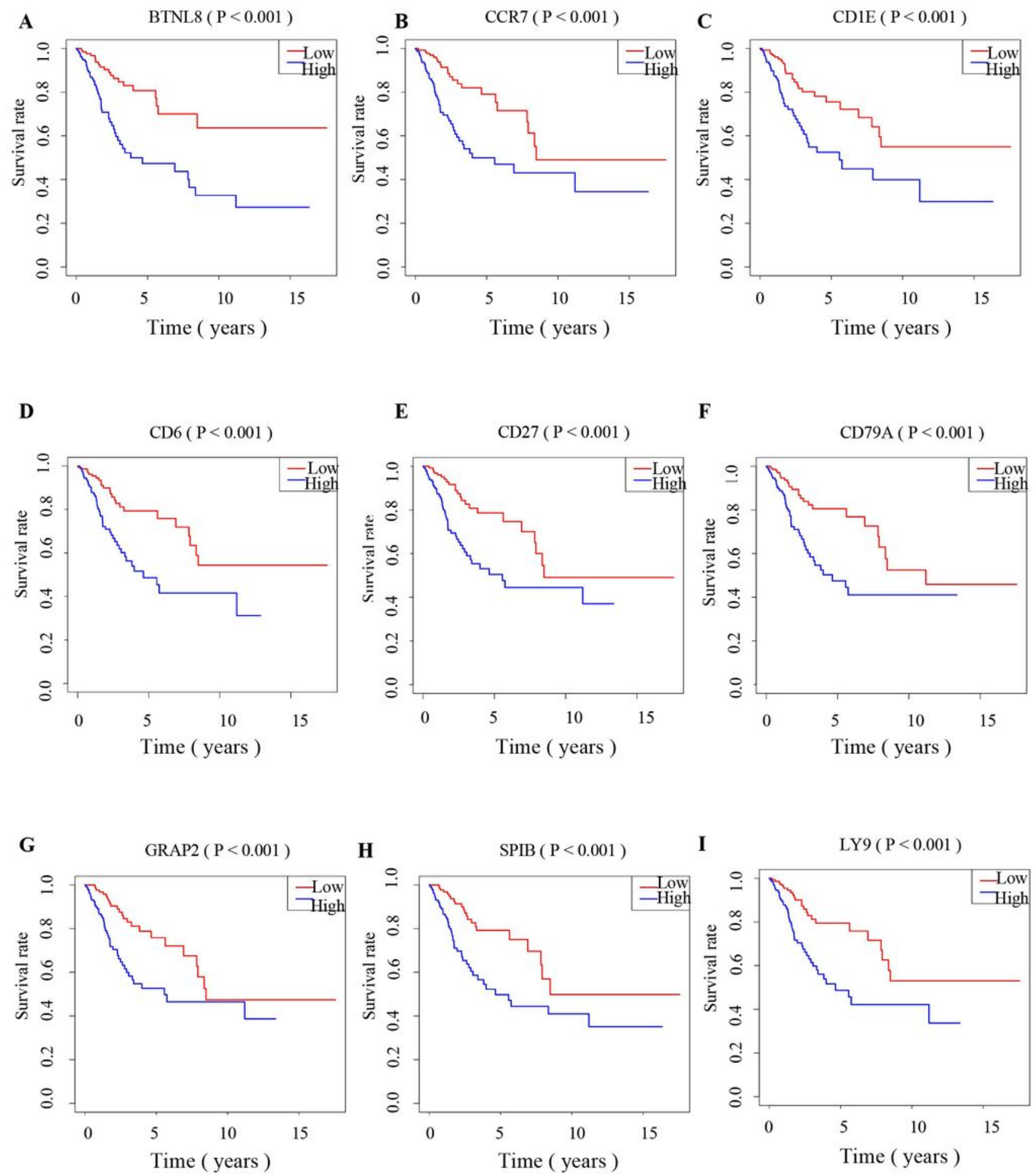

\section{Figure 5}

Correlation between expression of DEGs and overall survival of patient with cervical cancer based on TCGA datasets. Kaplan-Meier survival curves were performed for the representative DEGs. 